\title{
Laguerre and Hermite Collocation Methods for Unbounded Domains
}

\author{
Chao Zhang ${ }^{1, *}$ Hanfeng Yao $^{2}$ and Michael D. Samson ${ }^{3}$ \\ ${ }^{1}$ School of Mathematics and Statistics, Jiangsu Normal University, 221116, \\ China. \\ 2 School of Basic Education, Qingdao Huanghai University, 266427, China. \\ ${ }^{3}$ Department of Mathematics, DigiPen Institute of Technology, 637371, \\ Singapore.
}

Received 28 June 2016; Accepted (in revised version) 31 July 2017.

\begin{abstract}
We discuss spectral collocation methods based on Jacobi-Gauss-Lobatto points and Laguerre and Hermite collocation for unbounded domains. These methods are well conditioned, and some numerical experiments demonstrate quite high accuracy.
\end{abstract}

AMS subject classifications: 65M70, 41A05, 65L60, 34B40, 35L70

Key words: Collocation method, condition number, stability.

\section{Introduction}

The spectral collocation method that we consider approximates derivatives by direct differentiation of Lagrange interpolation polynomials at Gauss-type points. In applications involving non-linear problems or equations with variable coefficients, the performance of this approach is comparable to high-order finite difference methods and superior to the spectral method with modal basis functions. Nevertheless, it involves ill-conditioned linear systems when direct solvers and iteration methods exploiting a large number of collocation points are used. Various approaches to address this issue have been discussed. Coutsias and others $[4,5,8,16]$ proposed the integration preconditioning method, and Greengard [9] and El-Gendi [7] considered the spectral integration method. Wang et al. [23] introduced a Legendre (Chebyshev) collocation method based on a Birkhoff interpolation $[6,17]$ such that the corresponding system of linear equations is well-conditioned and the condition numbers do not depend on $N$, and their approach produces the exact inverse of the pseudospectral differentiation matrix of the highest derivative with only interior collocation points involved. The minimal eigenvalue of the second Jacobi pseudospectral differentiation matrix is a constant, so the differential operator of the highest order and the underlying boundary conditions can be associated with a suitable Birkhoff interpolation.

\footnotetext{
${ }^{*}$ Corresponding author. Email address: zcxz1977@163.com (C. Zhang) 
This opens the way to a new well-conditioned Jacobi-collocation method with another basis, to produce optimal integration preconditioners for usual collocation methods for exact boundary conditions. We provide such a new collocation scheme, where simulations for second order differential equations for example show both stability and high accuracy. However, numerical simulations in various areas of application (e.g. in quantum mechanics, biology, or financial mathematics) involve differential equations on unbounded domains, so the second part of this article is devoted to alternative Laguerre and Hermite collocation methods. Further, if the smallest eigenvalues of the corresponding matrices depend on their dimensions [24] such that these methods cannot be used, we construct new Birkhoff interpolation basis functions to produce both well-conditioned systems of linear algebraic equations and optimal preconditioners. The corresponding collocation schemes for second order differential equations with Dirichlet boundary conditions demonstrate high accuracy and improved stability. In Section 2, the interpolation basis uses JacobiGauss-Lobatto (JGL) points. In Section 3 and Section 4, Laguerre-Gauss-Radau (LGR) and Hermite-Gauss points are adopted for the construction of new bases. All of these bases are incorporated in the corresponding collocation schemes used in the numerical simulations. Our concluding remarks are in Section 5.

\section{Motivations and Observations from Collocation on a Finite Interval}

In this section, we extend the well-conditioned collocation method from Ref. [23] to general Jacobi-Gauss-type points. Our goal is to develop new collocation schemes for unbounded domains.

\subsection{Birkhoff interpolation basis on Jacobi-Gauss-Lobatto (JGL) points}

Let $\left\{h_{j}\right\}$ be the Lagrange interpolating polynomials associated with the JGL points $\left\{x_{j}\right\}_{j=0}^{N}$ where $x_{0}=-x_{N}=-1$ [19]. The differentiation matrices are defined as

$$
\boldsymbol{D}^{(k)}=\left(d_{i j}^{(k)}\right)_{0 \leq i, j \leq N}, \quad \boldsymbol{D}_{\mathrm{in}}^{(k)}=\left(d_{i j}^{(k)}\right)_{1 \leq i, j \leq N-1}, \quad d_{i j}^{(k)}=h_{j}^{(k)}\left(x_{i}\right),
$$

and we set $\boldsymbol{D}:=\boldsymbol{D}^{(1)}$ and $\boldsymbol{D}_{\text {in }}:=\boldsymbol{D}_{\text {in }}^{(1)}$. Explicit formulas for the entries of the matrix $\boldsymbol{D}$ and the relation are [19]

$$
\boldsymbol{D}^{(k)}=\boldsymbol{D} \boldsymbol{D} \cdots \boldsymbol{D}=\boldsymbol{D}^{k}, \quad k \geq 1 .
$$

It is also known that the condition numbers of the matrices $D_{\text {in }}^{(k)}$ grow like $N^{2 k}-$ cf. Refs. $[2,25]$. We are particularly interested in the second-order differentiation matrix. Weideman \& Trefethen [25] studied eigenvalues of the pseudospectral second derivative matrix with homogeneous Dirichlet boundary conditions, and observed that only about $2 N / \pi$ of the eigenvalues of the continuous operator are accurately approximated by the eigenvalues of the discrete operator. Vandeven [22] proposed a rigorous proof of that conjecture for the Galerkin Legendre spectral method, and Welfert [26] showed his results are also valid for the Legendre pseudospectral collocation method. Analogously, in the general Jacobi case the smallest eigenvalue of the matrix $-D_{\text {in }}^{(2)}$ can be approximated by $\pi^{2} / 4 \approx 2.467$. 
Table 1: Condition numbers, maximum and minimum absolute values of eigenvalues of $-D_{\text {in }}^{(2)}$ at JGL points with $\alpha=3, \beta=1$.

\begin{tabular}{c|c|c|c}
\hline$N$ & Cond. Number & Max|Eig| & Min|Eig| \\
\hline 32 & $4.00 E+03$ & $9.88 E+03$ & 2.47 \\
64 & $5.26 E+04$ & $1.30 E+05$ & 2.47 \\
128 & $7.61 E+05$ & $1.88 E+06$ & 2.47 \\
256 & $1.15 E+07$ & $2.86 E+07$ & 2.47 \\
\hline
\end{tabular}

For example, let us consider the JGL collocation scheme for the following model problem:

$$
-u^{\prime \prime}(x)=\lambda u(x), x \in I=(-1,1) \text { subject to } u( \pm 1)=0,
$$

which has the eigenvalues $\lambda_{j}=j^{2} \pi^{2} / 4, j=1,2, \cdots$. The corresponding discrete eigenvalue problem has the form $-\boldsymbol{D}_{\text {in }}^{(2)} \boldsymbol{u}=\tilde{\lambda} \boldsymbol{u}$, where the $(N-1)$ column vector $\boldsymbol{u}$ represents the approximation of the eigenvector $u(x)$ at the interior JGL points. We expect that about $2 N / \pi$ of the exact eigenvalues will be approximated by the corresponding discrete eigenvalues up to two-digit accuracy. Table 1 contains the absolute values of the largest and smallest eigenvalues of the matrix $-D_{\text {in }}^{(2)}$ obtained at the JGL points for $\alpha=3$ and $\beta=1$. These values show a near constant behaviour for the smallest eigenvalue and $N^{4}$-growth for the other. We point out that the constant behaviour of the smallest eigenvalue is crucial for the well-conditioning of the Legendre and Chebyshev collocation schemes with the Birkhoff interpolation. Similar ideas are used in the general Jacobi case.

Let $\mathscr{P}_{N}$ denote the space of polynomials of degree at most $N$. We seek basis polynomials $B_{j} \in \mathscr{P}_{N}, 0 \leq j \leq N$ such that

$$
\begin{array}{ll}
B_{0}(-1)=1, & B_{0}^{\prime \prime}\left(x_{i}\right)=0, \quad 1 \leq i \leq N-1, \quad B_{0}(1)=0 \\
B_{j}( \pm 1)=0, & B_{j}^{\prime \prime}\left(x_{i}\right)=\delta_{i j}, \quad 1 \leq i, j \leq N-1 \\
B_{N}(-1)=0, & B_{0}^{\prime \prime}\left(x_{i}\right)=0, \quad 1 \leq i \leq N-1, \quad B_{N}(1)=1 .
\end{array}
$$

Similarly to Refs. $[3,23]$, the basis is associated with the Birkhoff interpolation problem viz. given $u \in C^{2}(I)$, find polynomial $p \in \mathscr{P}_{N}$ such that $p( \pm 1)=u( \pm 1)$ and $p^{\prime \prime}\left(x_{i}\right)=u^{\prime \prime}\left(x_{i}\right)$, $1 \leq i \leq N-1$. From Eqs. (2.1), the interpolation polynomial can be expressed as

$$
p(x)=p(-1) B_{0}(x)+\sum_{j=1}^{N-1} u^{\prime \prime}\left(x_{j}\right) B_{j}(x)+p(1) B_{N}(x),
$$

Unlike Lagrange and Hermite interpolations, in the Birkhoff interpolation there is at least one point where the function and derivative values are not interpolated consecutively [17]. We set

$$
\boldsymbol{B}^{(k)}:=\left(B_{i j}^{(k)}\right)_{0 \leq i, j \leq N}, \quad \boldsymbol{B}_{\mathrm{in}}^{(k)}:=\left(B_{i j}^{(k)}\right)_{1 \leq i, j \leq N-1}, \quad k \geq 0,
$$

where $B_{i j}^{(k)}:=B_{j}^{(k)}\left(x_{i}\right)$, and let $\boldsymbol{B}:=\boldsymbol{B}^{0}, \boldsymbol{B}_{\text {in }}:=\boldsymbol{B}_{\text {in }}^{0}$. The following results can be proved similarly to Ref. [23]. 
Proposition 2.1. The differentiation matrices arising in the Lagrange and Birkhoff interpolation satisfy the equations

$$
\begin{aligned}
& \boldsymbol{B}^{(k)}=\boldsymbol{D}^{(k)} \boldsymbol{B}=\boldsymbol{D}^{k} \boldsymbol{B}=\boldsymbol{D} \boldsymbol{B}^{(k)}, \quad k \geq 1, \\
& \boldsymbol{D}_{\text {in }}^{(2)} \boldsymbol{B}_{\text {in }}=\boldsymbol{I}_{N-1},
\end{aligned}
$$

where $\boldsymbol{I}_{N-1}$ is an identity matrix.

Proposition 2.2 (Birkhoff interpolation at the JGL points). Let $\left\{x_{j}\right\}_{j=0}^{N}$ and $\left\{\omega_{j}\right\}_{j=0}^{N}$ be the JGL points and weights defined by (A.11), (A.12) and (A.13), respectively. The Birkhoff interpolation basis polynomials $\left\{B_{j}\right\}_{j=1}^{N-1}$ have the form

$$
B_{j}(x)=\left(\frac{-2 \beta_{0 j}}{\gamma_{0}}+(2 \beta-\alpha+1) \frac{2 \beta_{1 j}}{3 \gamma_{1}}-\sum_{k=2}^{N-2} \beta_{k j} \frac{d_{x}^{-2} P_{k}^{\alpha, \beta}(1)}{\gamma_{k}}\right) \frac{x+1}{2}+\sum_{k=0}^{N-2} \beta_{k j} \frac{d_{x}^{-2} P_{k}^{\alpha, \beta}(x)}{\gamma_{k}},
$$

where $d_{x}^{-2} P_{k}^{\alpha, \beta}(x)$ is calculated in (A.9),

$$
\begin{aligned}
& \gamma_{k}=\frac{2^{\alpha+\beta+1} \Gamma(k+\alpha+1) \Gamma(k+\beta+1)}{(2 k+\alpha+\beta+1) k ! \Gamma(k+\alpha+\beta+1)}, \\
& \beta_{k j}=\left(P_{k}^{\alpha, \beta}\left(x_{j}\right)+Q_{1} P_{k}^{\alpha, \beta}(-1)+Q_{2} P_{k}^{\alpha, \beta}(1)\right) \omega_{j}, \quad k \geq 0,
\end{aligned}
$$

and

$$
\begin{aligned}
Q_{1} & =\frac{P_{N-1}^{\alpha, \beta}(1) P_{N}^{\alpha, \beta}\left(x_{j}\right)-P_{N}^{\alpha, \beta}(1) P_{N-1}^{\alpha, \beta}\left(x_{j}\right)}{P_{N-1}^{\alpha, \beta}(-1) P_{N}^{\alpha, \beta}(1)-P_{N-1}^{\alpha, \beta}(1) P_{N}^{\alpha, \beta}(-1)}, \\
Q_{2}= & \frac{P_{N}^{\alpha, \beta}(-1) P_{N-1}^{\alpha, \beta}\left(x_{j}\right)-P_{N-1}^{\alpha, \beta}(-1) P_{N}^{\alpha, \beta}\left(x_{j}\right)}{P_{N-1}^{\alpha, \beta}(-1) P_{N}^{\alpha, \beta}(1)-P_{N-1}^{\alpha, \beta}(1) P_{N}^{\alpha, \beta}(-1)} .
\end{aligned}
$$

Moreover, the derivative of the corresponding Birkhoff polynomial is

$$
B_{j}^{\prime}(x)=\sum_{k=0}^{N-2} \beta_{k j} \frac{d_{x}^{-1} P_{k}^{\alpha, \beta}(x)}{\gamma_{k}}-\sum_{k=0}^{N-2} \beta_{k j} \frac{d_{x}^{-2} P_{k}^{\alpha, \beta}(1)}{2 \gamma_{k}},
$$

where $d_{x}^{-1} P_{k}^{\alpha, \beta}(x)$ is calculated in (A.8).

Remark 2.1. If $\alpha=\beta=0$, then Proposition 2.2 is the same as Proposition 3.5 in Ref. [23].

\subsection{Numerical results}

Example 2.1. Let us consider the boundary value problem

$$
-d_{x}^{2} u(x)+r(x) d_{x} u(x)+s(x) u(x)=f(x), x \in I \text { subject to } u( \pm 1)=u_{ \pm},
$$

where $r(x), s(x), f(x) \in C(I)$ are given functions. For brevity, we often denote the $k$-th derivative with respect to $x$-direction by $d_{x}^{k}$ hereafter. 
A collocation method for the problem (2.7) involves determining a polynomial $u_{N} \in \mathscr{P}_{N}(I)$ that satisfies the equations

$$
\left\{\begin{array}{l}
-d_{x}^{2} u_{N}\left(x_{i}\right)+r\left(x_{i}\right) d_{x} u_{N}\left(x_{i}\right)+s\left(x_{i}\right) u_{N}\left(x_{i}\right)=f\left(x_{i}\right), \quad 1 \leq i \leq N-1, \\
u_{N}( \pm 1)=u_{ \pm}
\end{array}\right.
$$

for a given set of points $x_{i}, 1 \leq i \leq N-1$. We consider three possible collocation methods.

(a) Jacobi-Lagrange collocation (JLCOL) method.

We represent the polynomial as

$$
u_{N}(x)=u_{-} h_{0}(x)+\sum_{j=1}^{N-1} u_{N}\left(x_{j}\right) h_{j}(x)+u_{+} h_{N}(x)
$$

and substitute this form into Eqs. (2.8) to produce the system of linear algebraic equations

$$
\left(-D_{\text {in }}^{(2)}+\Lambda_{r} D_{\text {in }}^{(1)}+\Lambda_{s}\right) \mathbf{u}=\mathbf{f}-\mathbf{u}_{B},
$$

where

$$
\begin{array}{ll}
\boldsymbol{\Lambda}_{r}=\operatorname{diag}\left(r\left(x_{1}\right), r\left(x_{2}\right), \cdots, r\left(x_{N-1}\right)\right), & \boldsymbol{\Lambda}_{s}=\operatorname{diag}\left(s\left(x_{1}\right), s\left(x_{2}\right), \cdots, s\left(x_{N-1}\right)\right), \\
\mathbf{u}=\left(u_{N}\left(x_{1}\right), u_{N}\left(x_{2}\right), \cdots, u_{N}\left(x_{N-1}\right)\right)^{\prime}, & \mathbf{f}=\left(f\left(x_{1}\right), f\left(x_{2}\right), \cdots, f\left(x_{N-1}\right)\right)^{\prime}, \\
\mathbf{u}_{B} \text { is the vector of }\left\{u_{-}\left(-d_{i 0}^{(2)}+r\left(x_{i}\right) d_{i 0}^{(1)}\right)+u_{+}\left(-d_{i N}^{(2)}+r\left(x_{i}\right) d_{i N}^{(1)}\right)\right\}_{i=1}^{N-1} .
\end{array}
$$

(b) Preconditioning collocation (PLCOL) method.

Property (2.4) allows us to use preconditioning for the ill-conditioned system (2.9), hence

$$
\boldsymbol{B}_{\text {in }}\left(-D_{\text {in }}^{(2)}+\Lambda_{r} D_{\text {in }}^{(1)}+\Lambda_{s}\right) \mathbf{u}=\boldsymbol{B}_{\text {in }}\left(\mathbf{f}-\mathbf{u}_{B}\right) .
$$

(c) New Jacobi-Birkhoff collocation (JBCOL) method.

We write the polynomial as

$$
u_{N}(x)=u_{-} B_{0}(x)+\sum_{j=1}^{N-1} v_{N}\left(x_{j}\right) B_{j}(x)+u_{+} B_{N}(x) .
$$

It follows from Eq. (2.2) that $v_{N}\left(x_{j}\right)=u_{N}^{\prime \prime}\left(x_{j}\right)$, and Eq. (2.8) takes the form

$$
\left(-\boldsymbol{I}_{N-1}+\Lambda_{r} B_{\text {in }}^{(1)}+\Lambda_{s} B_{\text {in }}\right) \mathbf{v}=\mathbf{f}-u_{-} \mathbf{v}_{-}-u_{+} \mathbf{v}_{+},
$$

where

$$
\begin{aligned}
& \mathbf{v}_{-}=\left\{-\frac{r\left(x_{i}\right)}{2}+s\left(x_{i}\right) \frac{1-x_{i}}{2}\right\}_{i=1}^{N-1}, \\
& \mathbf{v}_{+}=\left\{\frac{r\left(x_{i}\right)}{2}+s\left(x_{i}\right) \frac{1+x_{i}}{2}\right\}_{i=1}^{N-1} .
\end{aligned}
$$


Table 2: Condition numbers, accuracy and iterations for $\alpha=-1 / 2, \beta=3 / 2$.

\begin{tabular}{c|c|c|c|c|c|c|c|c|c}
\hline \multirow{2}{*}{$N$} & \multicolumn{3}{|c|}{ JLCOL (2.9) } & \multicolumn{3}{c|}{ PLCOL (2.10) } & \multicolumn{3}{c}{ JBCOL (2.11) } \\
\cline { 2 - 10 } & Cond & Error & Iters & Cond & Error & Iters & Cond & Error & Iters \\
\hline 64 & $7.97 \mathrm{E}+05$ & $3.63 \mathrm{E}-12$ & 201 & 5.15 & $3.42 \mathrm{E}-14$ & 7 & 6.43 & $3.23 \mathrm{E}-14$ & 10 \\
128 & $1.47 \mathrm{E}+07$ & $4.93 \mathrm{E}-11$ & 771 & 5.24 & $4.96 \mathrm{E}-13$ & 7 & 6.49 & $1.04 \mathrm{E}-13$ & 10 \\
256 & $3.26 \mathrm{E}+08$ & $4.96 \mathrm{E}-10$ & 4209 & 5.30 & $4.97 \mathrm{E}-13$ & 7 & 6.53 & $2.67 \mathrm{E}-13$ & 11 \\
512 & $7.33 \mathrm{E}+09$ & $2.19 \mathrm{E}-09$ & 27729 & 5.32 & $2.19 \mathrm{E}-13$ & 7 & 6.54 & $6.06 \mathrm{E}-13$ & 11 \\
1024 & $1.65 \mathrm{E}+11$ & $2.02 \mathrm{E}-08$ & 24151 & 5.33 & $2.02 \mathrm{E}-13$ & 7 & 6.55 & $4.83 \mathrm{E}-13$ & 11 \\
\hline
\end{tabular}

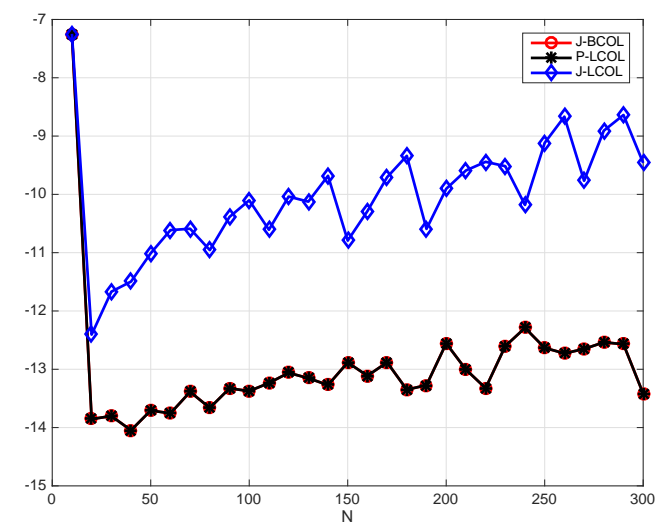

Figure 1: Maximum pointwise errors for $\alpha=-1 / 2, \beta=3 / 2$

Choosing $r(x)=-(1+\sin x), s(x)=e^{x}$ and the test function $u(x)=e^{\left(x^{2}-1\right) / 2}$, we find all three collocation methods converge. Table 2 contains condition numbers, errors and the number of iterations used in the BICGSTAB iterative method. The condition numbers of the new approach are independent of $N$ and it does not produce unacceptable round-off errors, even for a large number of collocation points. The graph in Fig. 1 displays the maximum pointwise errors for JBCOL, JLCOL and PLCOL for $\alpha=-1 / 2, \beta=3 / 2$.

\section{Birkhoff-Type Interpolation Functions Based on LGR Nodes}

Various problems on the semi-axis have attracted more attention recently. The Laguerre spectral and Laguerre collocation methods are popular approaches [10,11,13, 14, 18, 20], but these methods often produce ill-conditioned linear systems.

\subsection{Numerical observations}

Let $\left\{h_{j}\right\}$ be the Lagrange interpolating basis polynomials associated with the LGR points $\left\{x_{j}\right\}_{j=0}^{N}$ and consider

$$
\hat{h}_{j}(x)=e^{\left(x_{j}-x\right) / 2} h_{j}(x), \quad 0 \leq i, j \leq N,
$$


Table 3: Condition numbers, maximum and minimum absolute values of the eigenvalues of $\hat{D}_{\mathrm{in}}^{(2)}$.

\begin{tabular}{c|c|c|c}
\hline$N$ & Cond. Number & Max|Eig| & Min|Eig| \\
\hline 32 & $2.08 E+05$ & $1.10 E+02$ & $5.67 E-04$ \\
64 & $3.14 E+06$ & $4.28 E+02$ & $1.46 E-04$ \\
128 & $4.87 E+07$ & $1.69 E+03$ & $3.71 E-05$ \\
256 & $7.68 E+08$ & $6.69 E+03$ & $9.34 E-06$ \\
\hline
\end{tabular}

with the corresponding differentiation matrices

$$
\hat{\boldsymbol{D}}^{(k)}:=\left(d_{i j}^{(k)}\right)_{0 \leq i, j \leq N}, \quad \hat{\boldsymbol{D}}_{\mathrm{in}}^{(k)}:=\left(d_{i j}^{(k)}\right)_{1 \leq i, j \leq N},
$$

where $d_{i j}^{(k)}:=\hat{h}_{j}^{(k)}\left(x_{i}\right)$; and set $\hat{\boldsymbol{D}}:=\hat{\boldsymbol{D}}^{(1)}, \hat{\boldsymbol{D}}_{\text {in }}:=\hat{\boldsymbol{D}}_{\text {in }}^{(1)}$. Here we deal with the following eigenvalue problem:

$$
-u^{\prime \prime}(x)=\lambda u(x), \quad x \in \Lambda=(0, \infty), \quad u(0)=0 \text { and } \lim _{x \rightarrow \infty} u(x)=0 .
$$

Discretising this problem by the Laguerre-Lagrange collocation method, one only has to compute the eigenvalues of the matrix $-\hat{D}_{\text {in }}^{(2)}$, which are real and negative. As shown in Table 3 , the largest eigenvalue increases like $N^{2}$ and the smallest decreases like $N^{-2}$,consistent with theoretical analysis [24]. On the other hand, in the Legendre, Chebyshev and Jacobi collocation methods the smallest eigenvalues do not depend on $N$. However, on considering the matrices $\hat{D}_{\text {in }}^{(2)}-\mu I_{N}$ where $\mu$ is an arbitrary real number, the set of smallest eigenvalues is bounded by $|\mu|$ from above and the largest eigenvalues grow like $N^{2}$ such that the condition numbers of $\left(\hat{\boldsymbol{D}}_{\text {in }}^{(2)}-\mu I_{N}\right)^{-1}$ do not depend on $N$. We can construct a new basis for the solution of the second-order boundary value problems on the half line as follows.

\subsection{New Birkhoff-like basis on the half line}

Consider the set of functions

$$
\hat{\mathscr{P}}_{N}(\Lambda)=\operatorname{span}\left\{\hat{\mathscr{L}}_{0}(x), \hat{\mathscr{L}}_{1}(x), \cdots, \hat{\mathscr{L}}_{N}(x)\right\},
$$

where $\left\{\hat{\mathscr{L}}_{k}(x)\right\}_{k=0}^{N}$ are the Laguerre functions defined in Eq. (B.4). We consider the Birkhofftype interpolation problem - i.e. find a polynomial $\hat{p} \in \hat{\mathscr{P}}_{N}(\Lambda)$ such that for $u \in C^{2}(\Lambda)$, $\lim _{x \rightarrow \infty} u(x)=0$ the following relations hold:

$$
\begin{aligned}
& \hat{p}(0)=u(0), \\
& \hat{p}^{\prime \prime}\left(x_{i}\right)-\frac{1}{4} \hat{p}\left(x_{i}\right)=u^{\prime \prime}\left(x_{i}\right)-\frac{1}{4} u\left(x_{i}\right), \quad 1 \leq i \leq N .
\end{aligned}
$$

The Birkhoff interpolation function $\hat{p}$ for $u$ in Eqs. (3.4) can be represented uniquely in the form

$$
\hat{p}(x)=u(0) \hat{B}_{0}(x)+\sum_{j=1}^{N}\left(u^{\prime \prime}\left(x_{j}\right)-\frac{1}{4} u\left(x_{j}\right)\right) \hat{B}_{j}(x), \quad x \in \Lambda,
$$


after the polynomials $\left\{\hat{B}_{j}(x)\right\}_{j=0}^{N} \subseteq \hat{\mathscr{P}}_{N}(\Lambda)$ are determined such that

$$
\begin{array}{lll}
\hat{B}_{0}(0)=1, & \hat{B}_{0}^{\prime \prime}\left(x_{i}\right)-\frac{1}{4} \hat{B}_{0}\left(x_{i}\right)=0, & 1 \leq i \leq N, \\
\hat{B}_{j}(0)=0, & \hat{B}_{j}^{\prime \prime}\left(x_{i}\right)-\frac{1}{4} \hat{B}_{j}\left(x_{i}\right)=\delta_{i j}, & 1 \leq i, j \leq N .
\end{array}
$$

\subsection{Properties of the differentiation matrices}

Let us now discuss properties of the new basis functions used in the algorithm. Consider the matrices

$$
\hat{\boldsymbol{B}}^{(k)}:=\left(\hat{B}_{i j}^{(k)}\right)_{0 \leq i, j \leq N}, \quad \hat{\boldsymbol{B}}_{\mathrm{in}}^{(k)}:=\left(\hat{B}_{i j}^{(k)}\right)_{1 \leq i, j \leq N}, \quad k \geq 0,
$$

where $b_{i j}^{(k)}:=\hat{B}_{j}^{(k)}\left(x_{i}\right)$ and set $\hat{\boldsymbol{B}}:=\hat{\boldsymbol{B}}^{0}$, and $\hat{\boldsymbol{B}}_{\text {in }}:=\hat{\boldsymbol{B}}_{\text {in }}^{0}$. The following theorem plays an important role.

Theorem 3.1. Let $\widehat{B}_{j}(x)$ be the above-mentioned polynomials. Then we have the relation

$$
\left(\hat{D}_{\text {in }}^{(2)}-\frac{1}{4} I_{N}\right) \hat{\boldsymbol{B}}_{\text {in }}=I_{N}
$$

Proof. Since any polynomial $\phi \in \hat{\mathscr{P}}_{N}$ can be represented in the form

$$
\phi(x)=\sum_{p=0}^{N} \phi\left(x_{p}\right) \hat{h}_{p}(x),
$$

so that

$$
\phi^{(2)}(x)=\sum_{p=0}^{N} \phi\left(x_{p}\right) \hat{h}_{p}^{(2)}(x) .
$$

If $\phi=\hat{B}_{j}$ and $x=x_{i}, 1 \leq i, j \leq N$, then

$$
b_{i j}=\sum_{p=0}^{N} d_{i p} b_{p j}, \quad b_{i j}^{(2)}=\sum_{p=0}^{N} d_{i p}^{(2)} b_{p j} .
$$

It follows from (3.5) that $b_{0 j}=0$ for $1 \leq j \leq N$ and $b_{i j}^{(2)}-(1 / 4) b_{i j}=\delta_{i j}$ for $1 \leq i, j \leq N$. The equation (3.7) yields:

$$
\delta_{i j}=\sum_{p=1}^{N-1}\left(d_{i p}^{(2)}-\frac{1}{4} d_{i p}\right) b_{p j}, \quad 1 \leq i, j \leq N,
$$

so that $\left(\hat{\boldsymbol{D}}_{\text {in }}^{(2)}-(1 / 4) \boldsymbol{I}_{N}\right) \hat{\boldsymbol{B}}_{\text {in }}=\boldsymbol{I}_{N}$. 
Proposition 3.1. The Birkhoff interpolation basis functions $\left\{\hat{B}_{j}(x)\right\}_{j=0}^{N}$ that satisfy conditions (3.5) for the LGR nodes $x_{j}, j=0,1, \cdots, N$ are

$$
\begin{aligned}
\hat{B}_{0}(x)= & e^{-\frac{x}{2}} \\
\hat{B}_{j}(x)= & \hat{\omega}_{j} \sum_{k=0}^{N-1}\left(\hat{\mathscr{L}}_{k+1}\left(x_{j}\right)-\hat{\mathscr{L}}_{N}\left(x_{j}\right)\right) \hat{d}_{x}^{-2} \hat{\mathscr{L}}_{k}(x) \\
& -\hat{\omega}_{j}\left(\hat{\mathscr{L}}_{0}\left(x_{j}\right)-\hat{\mathscr{L}}_{N}\left(x_{j}\right)\right) \hat{d}_{x}^{-1} \hat{\mathscr{L}}_{0}(x), \quad 1 \leq j \leq N .
\end{aligned}
$$

where $\hat{\omega}_{j}=e^{x_{j}} \omega_{j}$ and $\hat{d}_{x}^{-2} \hat{\mathscr{L}}_{k}(x)$ is calculated using the formulas (B.8).

Proof. It is easily seen that (3.4) is equivalent to the Birkhoff polynomial interpolation problem: For $u \in C^{2}(\Lambda)$, find a polynomial $p \in \mathscr{P}_{N}(\Lambda)$ such that

$$
\left\{\begin{array}{l}
p(0)=u(0) \\
p^{\prime \prime}\left(x_{j}\right)-p^{\prime}\left(x_{j}\right)=u^{\prime \prime}\left(x_{j}\right)-u^{\prime}\left(x_{j}\right), \quad 1 \leq j \leq N
\end{array}\right.
$$

Note that if one can determine polynomials $\left\{B_{j}(x)\right\}_{j=0}^{N} \subseteq \mathscr{P}_{N}(\Lambda)$ such that

$$
\begin{aligned}
& B_{0}(0)=1, \quad B_{0}^{\prime \prime}\left(x_{i}\right)-B_{0}^{\prime}\left(x_{i}\right)=0, \quad 1 \leq i \leq N, \\
& B_{j}(0)=0, \quad B_{j}^{\prime \prime}\left(x_{i}\right)-B_{j}^{\prime}\left(x_{i}\right)=\delta_{i j}, \quad 1 \leq i, j \leq N \text {, }
\end{aligned}
$$

then the Birkhoff interpolation polynomial $p$ satisfying the relations (3.9) can be represented in the form

$$
p(x)=u(0) B_{0}(x)+\sum_{j=1}^{N}\left(u^{\prime \prime}\left(x_{j}\right)-u^{\prime}\left(x_{j}\right)\right) B_{j}(x), \quad x \in \Lambda .
$$

We take $B_{0}(x)=1$ for all $x \in \Lambda$ and assume that $B_{j}^{\prime \prime}(x)=\sum_{k=0}^{N-2} \beta_{k, j} \mathscr{L}_{k}(x)$ for $1 \leq j \leq N$. Then $B_{j}^{\prime}(x)=d_{x}^{-1} B_{j}^{\prime \prime}(x)-\beta_{-1, j}$ and using (B.7), we obtain

$$
B_{j}^{\prime \prime}(x)-B_{j}^{\prime}(x)=\sum_{k=0}^{N-2} \beta_{k, j}\left(\mathscr{L}_{k}(x)-\left(\mathscr{L}_{k}(x)-\mathscr{L}_{k+1}(x)\right)\right)+\beta_{-1, j}=\sum_{k=0}^{N-1} \beta_{k-1, j} \mathscr{L}_{k}(x) .
$$

We multiply the last equation by $\mathscr{L}_{m}(x) e^{-x}$ and integrate the result over $\Lambda$. It follows from (B.3), (B.5), and (3.10) that

$$
\left(B_{j}^{\prime \prime}(0)-B_{j}^{\prime}(0)\right) \omega_{0}+\mathscr{L}_{m}\left(x_{j}\right) \omega_{j}= \begin{cases}\beta_{m-1, j}, & m<N \\ 0, & m=N .\end{cases}
$$

If $m=N$, then

$$
B_{j}^{\prime \prime}(0)-B_{j}^{\prime}(0)=-\frac{\omega_{j}}{\omega_{0}} \mathscr{L}_{N}\left(x_{j}\right)=-\frac{1}{\mathscr{L}_{N}\left(x_{j}\right)}
$$


so that $\beta_{k, j}=\omega_{j}\left(\mathscr{L}_{k+1}\left(x_{j}\right)-\mathscr{L}_{N}\left(x_{j}\right)\right)$ for $-1 \leq k \leq N-2$, and

$$
B_{j}^{\prime}(x)=\omega_{j} \sum_{k=0}^{N-1}\left(\mathscr{L}_{k+1}\left(x_{j}\right)-\mathscr{L}_{N}\left(x_{j}\right)\right) d_{x}^{-1} \mathscr{L}_{k}(x)-\omega_{j}\left(\mathscr{L}_{0}\left(x_{j}\right)-\mathscr{L}_{N}\left(x_{j}\right)\right) .
$$

Since $\hat{B}_{j}(x)=e^{\left(x_{j}-x\right) / 2} B_{j}(x), 0 \leq i, j \leq N$, we can use (3.11) and obtain representations (3.8).

\subsection{New well-conditioned Laguerre collocation methods for second-order boundary value problems}

Let $r, s, f \in C(\Lambda)$. Consider the following boundary value problem:

$$
\left\{\begin{array}{l}
-d_{x}^{2} u(x)+r(x) d_{x} u(x)+s(x) u(x)=f(x), \quad x \in \Lambda, \\
u(0)=u_{0}, \quad u(x) \rightarrow 0 \text { as } x \rightarrow \infty
\end{array}\right.
$$

where $u_{0}$ is a constant.

\subsubsection{Numerical scheme}

Let $\left\{x_{i}\right\}_{i=0}^{N}$ be the set of LGR points. The collocation method for the problem (3.12) involves finding a polynomial $u_{N} \in \mathscr{P}_{N}(\Lambda)$ that satisfies the equations

$$
\left\{\begin{array}{l}
-d_{x}^{2} u_{N}\left(x_{i}\right)+r\left(x_{i}\right) d_{x} u_{N}\left(x_{i}\right)+s\left(x_{i}\right) u_{N}\left(x_{i}\right)=f\left(x_{i}\right), \quad 1 \leq i \leq N, \\
u(0)=u_{0}
\end{array}\right.
$$

(a) The Laguerre-Lagrange collocation (LLCOL) scheme.

We represent the approximate solution of Eqs. (3.12) as the polynomial

$$
u_{N}(x)=u_{0} \hat{h}_{0}(x)+\sum_{j=1}^{N} u_{N}\left(x_{j}\right) \hat{h}_{j}(x)
$$

and substitute it into Eqs. (3.13) so that

$$
\left(-\hat{\boldsymbol{D}}_{\mathrm{in}}^{(2)}+\Lambda_{r} \hat{\boldsymbol{D}}_{\mathrm{in}}^{(1)}+\Lambda_{s}\right) \mathbf{u}=\mathbf{f}-\mathbf{u}_{B},
$$

$$
\begin{aligned}
& \Lambda_{r}=\operatorname{diag}\left(r\left(x_{1}\right), r\left(x_{2}\right), \cdots, r\left(x_{N}\right)\right), \quad \Lambda_{s}=\operatorname{diag}\left(s\left(x_{1}\right), s\left(x_{2}\right), \cdots, s\left(x_{N}\right)\right), \\
& \mathbf{u}=\left(u_{N}\left(x_{1}\right), u_{N}\left(x_{2}\right), \cdots, u_{N}\left(x_{N}\right)\right)^{\prime}, \quad \mathbf{f}=\left(f\left(x_{1}\right), f\left(x_{2}\right), \cdots, f\left(x_{N}\right)\right)^{\prime}, \\
& \mathbf{u}_{B}=u_{0}\left(-\hat{h}_{0}^{\prime \prime}\left(x_{1}\right)+r\left(x_{i}\right) \hat{h}_{0}^{\prime}\left(x_{1}\right),-\hat{h}_{0}^{\prime \prime}\left(x_{2}\right)+r\left(x_{2}\right) \hat{h}_{0}^{\prime}\left(x_{2}\right), \cdots,-\hat{h}_{0}^{\prime \prime}\left(x_{N}\right)+r\left(x_{N}\right) \hat{h}_{0}^{\prime}\left(x_{N}\right)\right)^{\prime} .
\end{aligned}
$$

(b) The Laguerre-Birkhoff collocation (LBCOL) scheme.

Approximate solutions of the problem (3.12) are sought as linear combinations of the Birkhoff polynomials

$$
u_{N}(x)=u_{0} \hat{B}_{0}(x)+\sum_{j=1}^{N} v_{N}\left(x_{j}\right) \hat{B}_{j}(x)
$$


Table 4: Condition numbers of the systems (3.14).

\begin{tabular}{c|c|c|c|c}
\hline \multirow{2}{*}{} & \multicolumn{2}{|c|}{$\gamma=1$} & \multicolumn{2}{c}{$\gamma=5$} \\
\cline { 2 - 5 } & $\beta=1$ & $\beta=2$ & $\beta=1$ & $\beta=2$ \\
\hline$N=16$ & $3.05 E+1$ & $1.18 E+2$ & $6.89 E+0$ & $2.46 E+1$ \\
$N=32$ & $1.13 E+2$ & $4.47 E+2$ & $2.33 E+1$ & $9.04 E+1$ \\
$N=64$ & $4.35 E+2$ & $1.73 E+3$ & $8.78 E+1$ & $3.48 E+2$ \\
$N=128$ & $1.71 E+3$ & $6.84 E+3$ & $3.43 E+2$ & $1.36 E+3$ \\
$N=256$ & $6.79 E+3$ & $2.71 E+4$ & $1.36 E+3$ & $5.43 E+3$ \\
\hline
\end{tabular}

Table 5: Condition numbers of the systems (3.15).

\begin{tabular}{c|c|c|c|c|c|c}
\hline \multirow{2}{*}{} & \multicolumn{2}{|c|}{$\gamma=1$} & \multicolumn{2}{c|}{$\gamma=5$} & \multicolumn{2}{c}{$\gamma=10$} \\
\cline { 2 - 7 } & $\beta=1$ & $\beta=2$ & $\beta=1$ & $\beta=2$ & $\beta=1$ & $\beta=2$ \\
\hline$N=16$ & 3.88 & 1.00 & 17.2 & 4.81 & 30.0 & 9.25 \\
$N=32$ & 3.97 & 1.00 & 19.2 & 4.95 & 36.8 & 9.79 \\
$N=64$ & 3.99 & 1.00 & 19.8 & 4.99 & 39.1 & 9.94 \\
$N=128$ & 4.00 & 1.00 & 19.9 & 5.00 & 39.8 & 9.98 \\
$N=256$ & 4.00 & 1.00 & 19.9 & 4.99 & 39.9 & 9.99 \\
\hline
\end{tabular}

where $v_{N}\left(x_{j}\right)=u_{N}^{\prime \prime}\left(x_{j}\right)-(1 / 4) u_{N}\left(x_{j}\right)$, such that from (3.13) we have the form

$$
\left(-I_{N}+\Lambda_{r} \hat{\boldsymbol{B}}_{\text {in }}^{(1)}+\left(\Lambda_{s}-\frac{1}{4}\right) \hat{\boldsymbol{B}}_{\text {in }}\right) \mathbf{v}=\mathbf{f}-\mathbf{v}_{B}
$$

where $\mathbf{v}=\left(v\left(x_{1}\right), v\left(x_{2}\right), \cdots, v\left(x_{N}\right)\right)^{\prime}$.

\subsubsection{Numerical results}

If the solutions decay at infinity, a bounded interval can be chosen outside of which the solutions are negligible and the corresponding collocation points play no meaningful role. On the other hand, for solutions with non-negligible parts near the end of a bounded interval, one cannot expect to obtain a very good approximation. This means that the performance of the spectral methods in unbounded domains depends on whether we can find a suitable bounded interval and a set of collocation points where the largest is located near the endpoint of the chosen interval. In such computations, one often makes a linear transformation $y=\beta x[13,14,19,20]$, and instead of the problem (3.12) proceed to solve the related scaled form

$$
\left\{\begin{array}{l}
-\beta^{2} d_{y}^{2} v(y)+\beta r(y) d_{y} v(y)+s(y) v(y)=g(y), \quad y \in \Lambda, \\
v(0)=u_{0}, \quad v(y) \rightarrow 0 \text { as } y \rightarrow \infty
\end{array}\right.
$$

where $v(y)=u(\beta x), r(y)=r(\beta x), s(y)=(\beta x), g(y)=f(\beta x)$ and we write $d_{y}$ to denote differentiation with respect to the scaled variable $y$. 

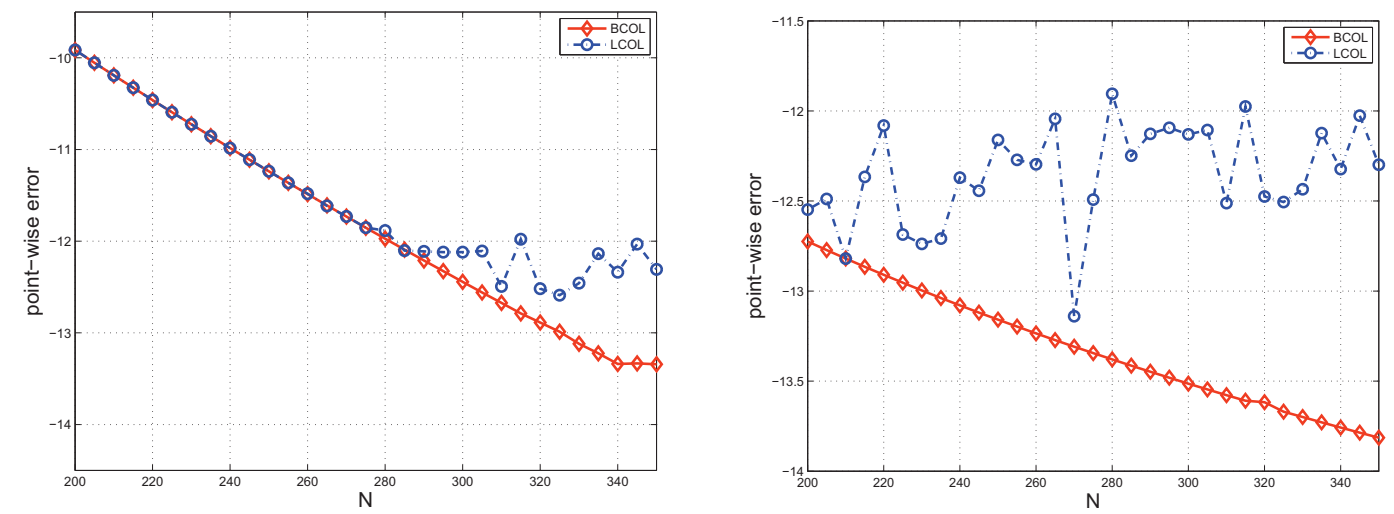

Figure 2: Comparison of maximum point-wise errors. Left: $\beta=1$; Right: $\beta=2$.

In our first example, we set $r(x)=0$ and $s(x)=\gamma$ in (3.12). Tables 4 and 5 show the condition numbers for the above two approaches. One can see that the condition numbers of the method (3.15) do not depend on $N$ and do not produce round-off errors. It is also notable that a suitable choice of parameter $\beta$ can improve the condition numbers. Secondly, we considered variable coefficients $r(x)=\sqrt{x}, s(x)=\ln (1+x)$ with the test function $u(x)=(1+x)^{-9 / 2}$. The graphs in Fig. 2 display the maximum point-wise errors for the LBCOL and LLCOL schemes. These numerical simulations illustrate the convergence of the collocation methods, the better stability of the method based on the Birkhoff interpolation polynomials, and also that a suitable choice of the scaling factor can improve results.

\section{Collocation Method Based on Hermite-Gauss Nodes}

The Hermite orthogonal approximation and Hermite-Gauss interpolation has provided satisfactory approximate solutions of problems defined on the real line $[1,12,15,20,27,28]$. In this section, we consider the Hermite collocation method based on Hermite-Gauss nodes.

\subsection{Numerical observations}

Let $\left\{h_{j}(x)\right\}_{j=0}^{N}$ be the Lagrange interpolating basis polynomials associated with the Hermite-Gauss nodes $\left\{x_{j}\right\}_{j=0}^{N}$ that are zeros of the Hermite polynomial $H_{N+1}(x)$, and let

$$
\tilde{h}_{j}(x):=e^{\left(x_{j}^{2}-x^{2}\right) / 2} h_{j}(x) .
$$

The pseudospectral differentiation matrices are defined as

$$
\tilde{\boldsymbol{D}}^{(k)}:=\left(d_{i j}^{(k)}\right)_{0 \leq i, j \leq N}, \quad d_{i j}^{(k)}=\tilde{h}_{j}^{(k)}\left(x_{i}\right) .
$$

In order to solve the eigenvalue problem

$$
-u^{\prime \prime}(x)=\lambda u(x)
$$


Table 6: Condition numbers, maximum and minimum absolute values of eigenvalues of $\tilde{\boldsymbol{D}}_{\text {in }}^{(2)}$.

\begin{tabular}{c|c|c|c}
\hline$N$ & Cond. Number & Max|Eig| & Min|Eig| \\
\hline 32 & $1.64 E+03$ & $6.05 E+01$ & $3.68 E-02$ \\
64 & $6.52 E+03$ & $1.23 E+02$ & $1.88 E-02$ \\
128 & $2.61 E+04$ & $2.49 E+02$ & $9.53 E-03$ \\
256 & $1.05 E+05$ & $5.02 E+02$ & $4.79 E-03$ \\
\hline
\end{tabular}

with boundary conditions decaying at the endpoints by the Hermite Lagrange collocation method, one has to find the eigenvalues of the matrix $-\tilde{D}^{(2)}$. Note that all eigenvalues of $-\tilde{D}^{(2)}$ obtained in numerical simulations are real and negative, with the smallest and largest eigenvalues shown in Table 6 . One can also see that the largest eigenvalue grows like $N$, and the smallest decreases like $N^{-1}$, consistent with theoretical analysis [24].

\subsection{Birkhoff-like interpolation based on Hermite-Gauss nodes}

Let $\Omega:=\{x \mid-\infty<x<\infty\}$, and let $x_{i}, i=0,1, \cdots, N$ be the Hermite-Gauss nodes. We look for the new basis functions $\tilde{B}_{j}(x)$ such that $\tilde{B}_{j}(x)$ tends to zero as $x$ tends to $\infty$ and

$$
\tilde{B}_{j}^{\prime \prime}\left(x_{i}\right)-x_{i}^{2} \tilde{B}_{j}\left(x_{i}\right)=\delta_{i j}, \quad 0 \leq j \leq N .
$$

Proposition 4.1. The Birkhoff interpolation basis functions $\left\{\tilde{B}_{j}(x)\right\}_{j=0}^{N}$ that are defined by the relations (4.1) for the Hermite-Gauss nodes $x_{i}, i=0,1, \cdots, N$ are

$$
\tilde{B}_{j}(x)=\sum_{k=0}^{N-1} \frac{\beta_{k, j}}{2 k+1} \tilde{H}_{k}(x), \quad 0 \leq j \leq N,
$$

where $\beta_{k, j}$ denote the coefficients in the expansion for the function $\tilde{h}_{j}(x)$, and $\tilde{H}_{k}(x)$ are defined in (C.1).

Note that the formulas (4.2) directly follow from Eq. (C.2).

\subsection{Hermite collocation methods for second-order boundary value problems}

Let $r, s, f \in C(\Omega)$. Consider the following boundary value problem:

$$
\left\{\begin{array}{l}
-d_{x}^{2} u(x)+r(x) d_{x} u(x)+s(x) u(x)=f(x), \quad x \in \Omega, \\
u(x) \rightarrow 0(|x| \rightarrow \infty)
\end{array}\right.
$$

We introduce the set

$$
\tilde{\mathscr{P}}_{N}(\Omega)=\operatorname{span}\left\{\tilde{H}_{0}(x), \tilde{H}_{1}(x), \cdots, \tilde{H}_{N}(x)\right\} .
$$

The Lagrange collocation method for the problem (2.7) involves finding a polynomial $u_{N} \in$ $\tilde{\mathscr{P}}_{N}(\Omega)$ such that

$$
-d_{x}^{2} u_{N}\left(x_{i}\right)+r\left(x_{i}\right) d_{x} u_{N}\left(x_{i}\right)+s\left(x_{i}\right) u_{N}\left(x_{i}\right)=f\left(x_{i}\right), \quad 0 \leq i \leq N .
$$


(a) The Hermite-Lagrange collocation (HLCOL) scheme.

On representing the numerical solution as

$$
u_{N}(x)=\sum_{j=0}^{N} u_{N}\left(x_{j}\right) \tilde{h}_{j}(x)
$$

and substituting this into Eq. (4.4), we obtain the system of linear algebraic equations

$$
\begin{gathered}
\left(-\tilde{\boldsymbol{D}}^{(2)}+\Lambda_{r} \tilde{\boldsymbol{D}}^{(1)}+\Lambda_{s}\right) \mathbf{u}=\mathbf{f}, \\
\tilde{\boldsymbol{D}}^{(1)}(i, j)=\tilde{h}_{j}^{\prime}\left(x_{i}\right), \quad \tilde{\boldsymbol{D}}^{(2)}(i, j)=\tilde{h}_{j}^{\prime \prime}\left(x_{i}\right), \quad 0 \leq i, j \leq N, \\
\Lambda_{r}=\operatorname{diag}\left(r\left(x_{0}\right), r\left(x_{1}\right), \cdots, r\left(x_{N}\right)\right), \quad \Lambda_{s}=\operatorname{diag}\left(s\left(x_{0}\right), s\left(x_{1}\right), \cdots, s\left(x_{N}\right)\right), \\
\mathbf{u}=\left(u_{N}\left(x_{0}\right), u_{N}\left(x_{1}\right), \cdots, u_{N}\left(x_{N}\right)\right)^{\prime}, \quad \mathbf{f}=\left(f\left(x_{0}\right), f\left(x_{1}\right), \cdots, f\left(x_{N}\right)\right)^{\prime} .
\end{gathered}
$$

(b) The Hermite-Birkhoff collocation (HBCOL) method.

When the numerical solution is represented as

$$
u_{N}(x)=\sum_{j=0}^{N} v_{N}\left(x_{j}\right) \tilde{B}_{j}(x)
$$

with $v_{N}\left(x_{j}\right):=u_{N}^{\prime \prime}\left(x_{j}\right)-x_{j}^{2} u_{N}\left(x_{j}\right)$, the corresponding system of linear algebraic equations (4.4) becomes

$$
\left(-I_{N+1}+\Lambda_{r} \tilde{\boldsymbol{B}}^{(1)}+\left(\Lambda_{s}-\Lambda_{x^{2}}\right) \tilde{\boldsymbol{B}}\right) \mathbf{v}=\mathbf{f},
$$

where $\Lambda_{x^{2}}=\operatorname{diag}\left(x_{0}^{2}, x_{1}^{2}, \cdots, x_{N}^{2}\right)$ and $\mathbf{v}=\left(v_{N}\left(x_{1}\right), v_{N}\left(x_{2}\right), \cdots, v_{N}\left(x_{N}\right)\right)^{\prime}$. In the numerical experiments, we use the transformation $y=\beta x$ and instead of the problem (4.3) proceed to solve the related scaled form

$$
\left\{\begin{array}{l}
-\beta^{2} d_{y}^{2} v(y)+\beta r(y) d_{y} v(y)+s(y) v(y)=g(y), \quad y \in \Omega \\
v(y) \rightarrow 0(|y| \rightarrow \infty)
\end{array}\right.
$$

where $v(y)=u(\beta x), r(y)=r(\beta x), s(y)=s(\beta x), g(y)=f(\beta x)$ and $d_{y}$ again denotes differentiation with respect to the scaled variable $y$.

For example, we set $r(x)=0$ and $s(x)=\left(x^{2}-1\right) / 2$ in (4.3). Tables 7 and 8 show the condition numbers for the systems (4.5) and (4.6), and it is notable that the condition numbers of (4.6) do not depend on $N$. Fig. 3 shows the maximum pointwise errors for the HBCOL and HLCOL methods, on adopting the test function $u(x)=\left(1+x^{2}\right)^{-9 / 2}$. One can see that the HBCOL method is free of round-off errors even for a large number of collocation points, whereas the condition numbers of the HLCOL systems mildly grow. Again, an appropriate choice of the parameter $\beta$ produced better numerical results. 
Table 7: Condition numbers of scheme (4.5).

\begin{tabular}{l|c|c|c|c|c}
\hline & $N=32$ & $N=64$ & $N=128$ & $N=256$ & $N=512$ \\
\hline$\beta=1$ & $2.96 E+2$ & $5.98 E+2$ & $1.20 E+3$ & $2.43 E+3$ & $4.89 E+3$ \\
$\beta=2$ & $1.06 E+2$ & $2.14 E+2$ & $4.31 E+2$ & $8.67 E+2$ & $1.74 E+3$ \\
\hline
\end{tabular}

Table 8: Condition numbers of scheme (4.6).

\begin{tabular}{l|c|c|c|c|c}
\hline & $N=32$ & $N=64$ & $N=128$ & $N=256$ & $N=512$ \\
\hline$\beta=1$ & 5.65 & 5.70 & 5.72 & 5.73 & 5.73 \\
$\beta=2$ & 2.27 & 2.28 & 2.29 & 2.29 & 2.29 \\
\hline
\end{tabular}
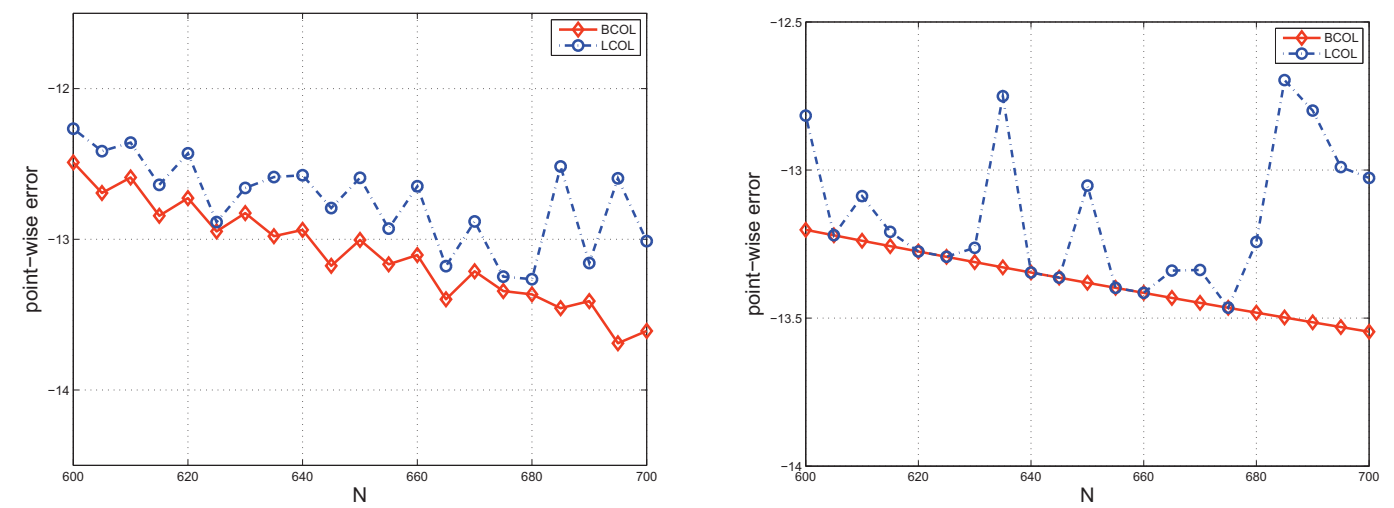

Figure 3: Comparison of maximum point-wise errors. Left: $\beta=1$; Right: $\beta=2$.

\section{Concluding Remarks}

New well-conditioned collocation methods have been considered. If minimal absolute eigenvalues have constant behaviour, the approach of Wang et al. [23] rendered a new Jacobi-Birkhoff collocation method that produces well-conditioned linear systems. However, since this method is not appropriate for unbounded domains, we introduced a new basis to produce well-conditioned linear algebraic systems. The stability and high accuracy of these methods were illustrated by some numerical simulations, and may also be applied to problems involving higher order differential equations.

\section{A. Jacobi Polynomials and Jacobi-Gauss-Lobatto Quadrature}

In this section, we revisit Jacobi polynomials and Jacobi-Gauss-Lobatto quadratures.

Let $P_{n}^{(\alpha, \beta)}(x)(x \in[-1,1]$ and $\alpha, \beta>-1)$ be the normalised Jacobi polynomials of the degree $n-$ cf. Ref. [21]. According to the formulas (3.110) in Ref. [19], they can be 
generated by the three-term recurrence relations

$$
\begin{aligned}
& P_{k+1}^{\alpha, \beta}(x)=\left(a_{k}^{\alpha, \beta} x-b_{k}^{\alpha, \beta}\right) P_{k}^{\alpha, \beta}(x)-c_{k}^{\alpha, \beta} P_{k-1}^{\alpha, \beta}(x), \quad k \geq 1, \\
& P_{0}^{\alpha, \beta}(x)=1, \quad P_{1}^{\alpha, \beta}(x)=\frac{1}{2}(\alpha+\beta+2) x+\frac{1}{2}(\alpha-\beta),
\end{aligned}
$$

where

$$
\begin{aligned}
a_{k}^{\alpha, \beta} & =\frac{(2 k+\alpha+\beta+1)(2 k+\alpha+\beta+2)}{2(k+1)(k+\alpha+\beta+1)}, \\
b_{k}^{\alpha, \beta} & =\frac{\left(\beta^{2}-\alpha^{2}\right)(2 k+\alpha+\beta+1)}{2(k+1)(k+\alpha+\beta+1)(2 k+\alpha+\beta)}, \\
c_{k}^{\alpha, \beta} & =\frac{(k+\alpha)(k+\beta)(2 k+\alpha+\beta+2)}{(k+1)(k+\alpha+\beta+1)(2 k+\alpha+\beta)} .
\end{aligned}
$$

It is also well-known that these polynomials form a complete orthogonal system in $L_{\omega^{\alpha, \beta}}^{2}(I)$ - i.e.

$$
\int_{-1}^{1} P_{k}^{\alpha, \beta}(x) P_{l}^{\alpha, \beta}(x) \omega^{\alpha, \beta}(x) d x=\gamma_{k}^{\alpha, \beta} \delta_{k l},
$$

where $\gamma_{k}^{\alpha, \beta}=\left\|P_{k}^{\alpha, \beta}\right\|_{\omega^{\alpha, \beta}}^{2}$. Moreover,

$$
P_{k}^{\alpha, \beta}(x)=(-1)^{k} P_{k}^{\beta, \alpha}(-x), \quad P_{k}^{\alpha, \beta}(1)=\frac{\Gamma(k+\alpha+1)}{k ! \Gamma(\alpha+1)}
$$

and

$$
P_{k}^{\alpha, \beta}(x)=A_{k}^{\alpha, \beta} d_{x} P_{k-1}^{\alpha, \beta}(x)+B_{k}^{\alpha, \beta} d_{x} P_{k}^{\alpha, \beta}(x)+C_{k}^{\alpha, \beta} d_{x} P_{k+1}^{\alpha, \beta}(x), \quad k \geq 1,
$$

with

$$
\begin{aligned}
A_{k}^{\alpha, \beta} & =-\frac{2(k+\alpha)(k+\beta)}{(k+\alpha+\beta)(2 k+\alpha+\beta)(2 k+\alpha+\beta+1)}, \\
B_{k}^{\alpha, \beta} & =\frac{2(\alpha-\beta)}{(2 k+\alpha+\beta)(2 k+\alpha+\beta+2)}, \\
C_{k}^{\alpha, \beta} & =\frac{2(k+\alpha+\beta+1)}{(2 k+\alpha+\beta+1)(2 k+\alpha+\beta+2)} .
\end{aligned}
$$

If $d_{x}^{-m}, m>0$ denotes the integral operator

$$
\begin{aligned}
& d_{x}^{-1} u(x):=\int_{-1}^{x} u(t) d t \\
& d_{x}^{-m} u(x):=d_{x}^{-1}\left(d_{x}^{-(m-1)} u(x)\right), \quad m \geq 2,
\end{aligned}
$$


then (A.2) and (A.3) imply that

$$
\begin{aligned}
& d_{x}^{-1} P_{k}^{\alpha, \beta}(x)=A_{k}^{\alpha, \beta} P_{k-1}^{\alpha, \beta}(x)+B_{k}^{\alpha, \beta} P_{k}^{\alpha, \beta}(x)+C_{k}^{\alpha, \beta} P_{k+1}^{\alpha, \beta}(x)-H_{k}^{\alpha, \beta}, \quad k \geq 1, \\
& d_{x}^{-1} P_{0}^{\alpha, \beta}(x)=x+1,
\end{aligned}
$$

where

$$
H_{k}^{\alpha, \beta}=\left(A_{k}^{\alpha, \beta} P_{k-1}^{\alpha, \beta}(-1)+B_{k}^{\alpha, \beta} P_{k}^{\alpha, \beta}(-1)+C_{k}^{\alpha, \beta} P_{k+1}^{\alpha, \beta}(-1)\right)
$$

and the constants $A_{k}^{\alpha, \beta}, B_{k}^{\alpha, \beta}$ and $C_{k}^{\alpha, \beta}$ are defined in (A.4)-(A.6). In addition, one has

$$
\begin{aligned}
d_{x}^{-2} P_{k}^{\alpha, \beta}(x)= & A_{k}^{\alpha, \beta} A_{k-1}^{\alpha, \beta} P_{k-2}^{\alpha, \beta}(x)+\left(A_{k}^{\alpha, \beta} B_{k-1}^{\alpha, \beta}+A_{k}^{\alpha, \beta} B_{k}^{\alpha, \beta}\right) P_{k-1}^{\alpha, \beta}(x) \\
& +\left(A_{k}^{\alpha, \beta} C_{k-1}^{\alpha, \beta}+\left(B_{k}^{\alpha, \beta}\right)^{2}+A_{k+1}^{\alpha, \beta} C_{k}^{\alpha, \beta}\right) P_{k}^{\alpha, \beta}(x) \\
& +C_{k}^{\alpha, \beta}\left(B_{k}^{\alpha, \beta}+B_{k+1}^{\alpha, \beta}\right) P_{k+1}^{\alpha, \beta}(x)+C_{k}^{\alpha, \beta} C_{k+1}^{\alpha, \beta} P_{k+2}^{\alpha, \beta}(x) \\
& -H_{k}^{\alpha, \beta} x-\left(A_{k}^{\alpha, \beta} H_{k-1}^{\alpha, \beta}+B_{k}^{\alpha, \beta} H_{k}^{\alpha, \beta}+C_{k}^{\alpha, \beta} H_{k+1}^{\alpha, \beta}+H_{k}^{\alpha, \beta}\right)
\end{aligned}
$$

for $k \geq 2$, and

$$
\begin{aligned}
& d_{x}^{-2} P_{1}^{\alpha, \beta}(x)=\frac{(\alpha+\beta+2) x^{3}+3(\alpha-\beta) x^{2}+3(\alpha-3 \beta-2) x+(\alpha-5 \beta-4)}{12}, \\
& d_{x}^{-2} P_{0}^{\alpha, \beta}(x)=\frac{(1+x)^{2}}{2} .
\end{aligned}
$$

Next, we consider the Jacobi-Gauss-Lobatto (JGL) points $\left\{x_{j}\right\}_{j=0}^{N}, x_{0}=-1, x_{N}=1$ that are the zeros of the polynomials $\left(1-x^{2}\right) d_{x} P_{N}^{\alpha, \beta}(x)$, and the quadrature weights

$$
\begin{aligned}
& \omega_{0}=\frac{2^{\alpha+\beta+1}(\beta+1) \Gamma^{2}(\beta+1)(N-1) ! \Gamma(N+\alpha+1)}{\Gamma(N+\beta+1) \Gamma(N+\alpha+\beta+2)}, \\
& \omega_{N}=\frac{2^{\alpha+\beta+1}(\alpha+1) \Gamma^{2}(\alpha+1)(N-1) ! \Gamma(N+\beta+1)}{\Gamma(N+\alpha+1) \Gamma(N+\alpha+\beta+2)}, \\
& \omega_{j}=\frac{1}{\left(1-x_{j}^{2}\right)^{2}} \frac{\tilde{G}_{N-2}^{\alpha+1, \beta+1}}{\left[d_{x} P_{N-1}^{\alpha+1, \beta+1}\left(x_{j}\right)\right]^{2}}, \quad 1 \leq j \leq N-1,
\end{aligned}
$$

where

$$
\tilde{G}_{N}^{\alpha, \beta}=\frac{2^{\alpha+\beta+1} \Gamma(N+\alpha+2) \Gamma(N+\beta+2)}{(N+1) ! \Gamma(N+\alpha+\beta+2)} .
$$

Then

$$
\int_{-1}^{1} \phi(x) \omega(x) d x=\sum_{j=0}^{N} \phi\left(x_{j}\right) \omega_{j}
$$

for any $\phi \in \mathscr{P}_{2 N-1}(I)$. 


\section{B. Laguerre Functions and Laguerre-Gauss-Radau Quadrature}

The Laguerre polynomials defined by

$$
\mathscr{L}_{k}(x)=\frac{1}{k !} e^{x} d_{x}^{k}\left(x^{k} e^{-x}\right), \quad k \geq 0, x \in \Lambda,
$$

satisfy the relations

$$
\begin{aligned}
& \mathscr{L}_{k}(0)=1, \\
& \mathscr{L}_{k}(x)=d_{x} \mathscr{L}_{k}(x)-d_{x} \mathscr{L}_{k+1}(x), \quad k \geq 0, \\
& -x d_{x} \mathscr{L}_{k}(x)=k \mathscr{L}_{k-1}(x)-k \mathscr{L}_{k}(x), \quad k \geq 1,
\end{aligned}
$$

and are orthogonal with the weight function $e^{-x}[19,21]-$ i.e.

$$
\int_{\Lambda} \mathscr{L}_{k}(x) \mathscr{L}_{l}(x) e^{-x} d x=\delta_{k l}
$$

We also define the corresponding Laguerre function as

$$
\hat{\mathscr{L}}_{k}(x)=e^{-\frac{x}{2}} \mathscr{L}_{k}(x), \quad k \geq 0,
$$

consider the Laguerre-Gauss-Radau nodes $\left\{x_{j}\right\}_{j=0}^{N}$ that are the zeros of the polynomials $x d_{x} \mathscr{L}_{N+1}(x)$, and define the Christoffel numbers

$$
\omega_{j}=\frac{1}{(N+1) \mathscr{L}_{N}^{2}\left(x_{j}\right)}, \quad 0 \leq j \leq N .
$$

According to formulas (7.33) of Ref. [19], for any $p \in \mathscr{P}_{2 N}$ the relation

$$
\int_{\Lambda} p(x) e^{-x} d x=\sum_{j=0}^{N} p\left(x_{j}\right) \omega_{j}
$$

holds. If $d_{x}^{-m}$ and $\hat{d}_{x}^{-m}, m>0$ denote the operators

$$
\begin{array}{ll}
d_{x}^{-1} \mathscr{L}_{k}(x):=\int_{0}^{x} \mathscr{L}_{k}(t) d t, & d_{x}^{-2} \mathscr{L}_{k}(x):=d_{x}^{-1}\left(d_{x}^{-1} \mathscr{L}_{k}(x)\right), \\
\hat{d}_{x}^{-1} \hat{\mathscr{L}}_{k}(x):=e^{-\frac{x}{2}} d_{x}^{-1} \mathscr{L}_{k}(x), & \hat{d}_{x}^{-2} \hat{\mathscr{L}}_{k}(x):=e^{-\frac{x}{2}} d_{x}^{-2} \mathscr{L}_{k}(x),
\end{array}
$$

then (B.1) implies

$$
\begin{aligned}
& d_{x}^{-1} \mathscr{L}_{k}(x)=\mathscr{L}_{k}(x)-\mathscr{L}_{k+1}(x) \\
& d_{x}^{-2} \mathscr{L}_{k}(x)=\mathscr{L}_{k}(x)-2 \mathscr{L}_{k+1}(x)+\mathscr{L}_{k+2}(x)
\end{aligned}
$$

Similarly,

$$
\begin{aligned}
& \hat{d}_{x}^{-1} \hat{\mathscr{L}}_{k}(x)=\hat{\mathscr{L}}_{k}(x)-\hat{\mathscr{L}}_{k+1}(x), \\
& \hat{d}_{x}^{-2} \hat{\mathscr{L}}_{k}(x)=\hat{\mathscr{L}}_{k}(x)-2 \hat{\mathscr{L}}_{k+1}(x)+\hat{\mathscr{L}}_{k+2}(x) .
\end{aligned}
$$




\section{Hermite Functions}

Let $\Omega=(-\infty, \infty)$. The Hermite polynomials are defined by

$$
H_{k}(x):=(-1)^{k} e^{x^{2}} d_{x}^{k}\left(e^{-x^{2}}\right), \quad k \geq 0, x \in \Omega .
$$

The Hermite functions are defined by

$$
\tilde{H}_{k}(x):=\frac{1}{\pi^{\frac{1}{4}} \sqrt{2^{k} k !}} e^{-x^{2} / 2} H_{k}(x), \quad k \geq 0,
$$

and satisfy the differential equation

$$
\tilde{H}_{k}^{\prime \prime}(x)+\left(2 k+1-x^{2}\right) \tilde{H}_{k}(x)=0, \quad k \geq 0 .
$$

\section{Acknowledgments}

The work is supported in part by NSF of China N.11571151 and N.11371123, Research Fund for Young Teachers of Jiangsu Normal University N.11XLR27, and Priority Academic Program Development of Jiangsu Higher Education Institutions. The majority of this joint work was carried out during the second author's visit to the Nanyang Technological University, and the second author would like to thank Prof. Li-Lian Wang for his hospitality and financial support.

\section{References}

[1] J.P. Boyd, The rate of convergence of Hermite function series, Math. Comp. 35, 1309-1316 (1980).

[2] C. Canuto, High-order methods for PDEs: recent advances and new perspectives, in 6th International Congress on Industrial and Applied Mathematics, pp. 57-87, European Mathematical Society (2009).

[3] F.A. Costabile and E. Longo, A Birkhoff interpolation problem and application, Calcolo 47, 4963 (2010).

[4] E. Coutsias, T. Hagstrom, J.S. Hesthaven, and D. Torres, Integration preconditioners for differential operators in spectral $\tau$-methods, in Proceedings of the Third International Conference on Spectral and High Order Methods, A. Ilin and R. Scott (Eds.), pp. 21-38, Houston Journal of Mathematics (1996).

[5] E.A. Coutsias, T. Hagstrom, and D. Torres, An efficient spectral method for ordinary differential equations with rational function coefficients, Math. Comp. 65, 611-635 (1996).

[6] P.J. Davis, Interpolation and Approximation, Dover (1975).

[7] S.E. El-Gendi, Chebyshev solution of differential, integral and integro-differential equations, Comput. J. 12, 282-287 (1969).

[8] M.E. Elbarbary, Integration preconditioning matrix for ultraspherical pseudospectral operators, SIAM J. Sci. Comput. 28, 1186-1201 (2006).

[9] L. Greengard, Spectral integration and two-point boundary value problems, SIAM J. Numer. Anal. 28, 1071-1080 (1991). 
[10] B.Y. Guo and J. Shen, Laguerre-Galerkin method for nonlinear partial differential equations on a semi-infinite interval, Numer. Math. 86, 635-654 (2000).

[11] B.Y. Guo, J. Shen, and C.L. Xu, Generalized Laguerre approximation and its applications to exterior problems, J. Comp. Math. 23, 113-130 (2005).

[12] B.Y. Guo, J. Shen, and C.L. Xu, Spectral and pseudospectral approximations using Hermite functions: Application to the Dirac equation, Adv. Comput. Math. 19, 35-55 (2003).

[13] B.Y. Guo, T. Sun, and C. Zhang, Jacobi and Laguerre quasi-orthogonal approximations and related interpolation, Math. Comp. 82, 413-441 (2013).

[14] B.Y. Guo, L.L Wang, and Z.Q Wang, Generalized Laguerre interpolation and pseudospectral method for unbounded domains, SIAM J. Numer. Anal. 43, 2567-2589 (2006).

[15] B.Y. Guo and C.L. Xu, Hermite pseudospectral method for nonlinear partial differential equations, Math. Model. Numer. Anal. 34, 859-872 (2000).

[16] J. Hesthaven, Integration preconditioning of pseudospectral operators I. Basic linear operators, SIAM J. Numer. Anal. 35, 1571-1593 (1998).

[17] G.G. Lorentz, K. Jetter, and S.D. Riemenschneider, Birkhoff Interpolation, Encyclopedia of Mathematics and its Applications, 19 Addison-Wesley (1983).

[18] J. Shen. Stable and efficient spectral methods in unbounded domains using Laguerre functions, SIAM J. Numer. Anal. 38, 1113-1133 (2000).

[19] J. Shen, T. Tang, and L.L. Wang, Spectral Methods: Algorithms, Analysis and Applications, Series in Computational Mathematics, 41, Springer (2011).

[20] J. Shen and L.L. Wang, Some recent advances in spectral methods for unbounded domains, Comm. in Comp. Phys. 5, 195-141 (2009).

[21] G. Szegö, Orthogonal Polynomials, AMS Colloquium Publication, 23, American Mathematical Society (1975).

[22] H. Vandeven, On the eigenvalues of second-order spectral differentiation operators, Comput. Methods Appl. Mech. Engrg. 80, 313-318 (1990).

[23] L.L. Wang, M.D. Samson, and X.D. Zhao, A well-conditioned collocation method using a pseudospectral integration matrix, SIAM J. Sci. Comput. 59, 667-687 (2014).

[24] J.A.C. Weideman, The eigenvalues of Hermite and rational spectral differentiation matrices, Numer. Math. 61, 409-431 (1992).

[25] J.A.C. Weideman and L.N. Trefethen, The eigenvalues of second-order spectral differentiation matrices, SIAM J. Numer. Anal. 25, 1279-1298 (1988).

[26] B.D. Welfert, On the eigenvalues of second-order pseudospectral differentiation operators, Comput. Methods Appl. Mech. Engrg. 116, 281-292 (1994).

[27] X.M. Xiang and Z.Q. Wang, Generalized Hermite spectral method and its applications to problems in unbounded domains, SIAM J. Numer. Anal. 48, 1231-1253 (2010).

[28] C. Zhang and B.Y. Guo, Generalized Hermite spectral method matching asymptotic behaviors, J. Comp. Appl. Math. 255, 616-634 (2014). 